\title{
SELECCIÓN DE NUEVAS ESPECIES PARA ZONAS CON DÉFICIT HÍDRICO
}

\author{
Braulio Gutiérrez ${ }^{1}$, María Paz Molina ${ }^{1}$ y Santiago Barros ${ }^{2}$
}

\section{RESUMEN}

Se evalúan preliminarmente los resultados de tres ensayos de especies y progenies de las especies Eucalyptus camaldulensis, E. cladocalyx, E. diversicolor, E. sideroxylon, E. tereticornis, E. tricarpa y Corymbia maculata, a dos y tres años de edad en la Región de Coquimbo, y de tres ensayos clonales de Eucalyptus camaldulensis a 10 meses de edad en las Regiones de Coquimbo y Valparaíso.

Se trata de resultados iniciales que requerirán complementarse con análisis efectuados a una mayor edad, para continuar con un programa de mejoramiento genético en el caso de especies y progenies y para confirmar el desempeño del mejor material para programas de forestación en el caso de los clones.

Las especies Eucalyptus camaldulensis, E. cladocalyx, E. sideroxylon y E. tereticornis, confirman experiencias anteriores, respecto de ser adecuadas para la forestación en zonas semiáridas, en especial si se piensa en madera para usos menores y energía, en protección de suelos, en producción melífera y en sistemas integrados, agroforestales o silvopastorales.

De acuerdo a la tendencia inicial de los ensayos clonales, Eucalytus. camaldulensis exhibe crecimientos a los 10 meses de edad, similares a los obtenidos a los dos años en ensayos con plantas de semillas y con un menor grado de selección. Esta situación representa un alentador resultado para el programa de mejoramiento genético y confirma una vez más el interés de esta especie para la zona semiárida.

Palabras clave: Eucaliptos, ensayos especies progenies, ensayos clonales, zonas semiáridas. 


\section{SUMMARY}

Early results from three two and three years old species-progeny trials, including Eucalyptus camaldulensis, E. cladocalyx, E. diversicolor, E. sideroxylon, E. tereticornis, E. tricarpa and Corymbia maculata, in the Coquimbo Region, and three ten months old E. camaldulensis clonal trials, in the Coquimbo and Valparaíso Regions, are evaluated.

The research requires a longer evaluation time in order to develop an improvement program with the best species and progenies and to confirm the better genetic material for afforestation programs in the case of the clonal trials.

Eucalyptus camaldulensis, E. cladocalyx, E. sideroxylon y E. tereticornis, as seen in previous experiences, are appropriated species to use in the semiarid zone, especially if final expected products are wood for minor uses and energy, or the objectives are soil protection, agroforestry and honey production.

Accordingly to the clonal trials preliminary trends, E. camaldulensis shows at ten months old results equivalent to others ones previously obtained at two years old in trials with a minor degree of genetic selection. That represents so far a success for the breeding program and confirms once more the value of the species to be used in the Chilean semiarid zone.

Keywords: Eucalypts, species progeny trials, clonal trials, semiarid zones 


\section{INTRODUCCIÓN}

Desde los inicios del Instituto Forestal en el año 1962, se ha trabajado permanentemente en la introducción y evaluación de especies para la forestación en diferentes regiones del país, desde aquella época, en la que prácticamente la única especie empleada en los planes de forestación era pino radiata (Pinus radiata) y la superficie plantada en el país era probablemente inferior a 200 mil hectáreas, la situación ha cambiado fuertemente, hoy existen 2,3 millones de hectáreas de plantaciones forestales y, aunque pino radiata sigue siendo la principal especie, se ha iniciado una progresiva diversificación.

Información a diciembre del año 2008 indica que la superficie de plantaciones a través de las regiones del país está compuesta como se indica en el Cuadro № 1.

\section{Cuadro $\mathrm{N}^{\circ} 1$}

\section{SUPERFICIE PLANTACIONES FORESTALES SEGÚN ESPECIE Y REGIÓN}

\begin{tabular}{|c|c|c|c|c|c|c|c|c|c|c|c|c|}
\hline REGIÓN & $\begin{array}{l}\text { Pinus } \\
\text { radiata }\end{array}$ & $\begin{array}{c}\text { Eucalyptus } \\
\text { globulus }\end{array}$ & $\begin{array}{c}\begin{array}{c}\text { Eucalyptus } \\
\text { nitens }\end{array} \\
\end{array}$ & $\begin{array}{l}\text { Atriplex } \\
\text { spp }\end{array}$ & $\begin{array}{c}\text { Pinus } \\
\text { ponderosa }\end{array}$ & \begin{tabular}{|c|}
$\begin{array}{c}\text { Eucalyptus } \\
\text { spp }\end{array}$ \\
\end{tabular} & $\begin{array}{l}\text { Prosopis } \\
\text { tamarugo }\end{array}$ & \begin{tabular}{|c|}
$\begin{array}{c}\text { Pseudotsuga } \\
\text { menzesii }\end{array}$ \\
\end{tabular} & $\begin{array}{c}\text { Populus } \\
\text { spp }\end{array}$ & $\begin{array}{c}\text { Prosopis } \\
\text { spp }\end{array}$ & Otras & TOTAL \\
\hline & \multicolumn{12}{|c|}{ (ha) } \\
\hline $\begin{array}{l}\text { Arica y Parinacota } \\
\text { Tarapacá }\end{array}$ & & 342 & & 32 & & & 20.639 & & & 3.246 & 684 & 24.943 \\
\hline Antolagasta & & 2 & & 44 & & & 47 & & & 302 & 861 & 1.256 \\
\hline Atacama & & 1.414 & & 32 & & & 12 & & & 488 & 1.480 & 3.426 \\
\hline Coquimbo & & 2.615 & & 58.985 & & 140 & & & & 1.139 & 20.242 & 83.121 \\
\hline Valparaiso & 10.070 & 40.018 & & & & 566 & & & 6 & & 915 & 51.575 \\
\hline Metropolitana & & 11.369 & & & & & & & 1 & 5 & 2.570 & 13.945 \\
\hline O?Higgins & 64.875 & 34.851 & 17 & & & 5 & & & 1.520 & & 323 & 101.591 \\
\hline Maule & 397.292 & 37.377 & 736 & & & 79 & & 217 & 2.731 & & 652 & 439.084 \\
\hline Bio Bio & 607.659 & 191.707 & 51.201 & & 179 & 6.775 & & 379 & 1.095 & & 2.253 & 861.248 \\
\hline La Araucania & 257.093 & 114.980 & 48.274 & & 1.891 & 3.927 & & 6.468 & 346 & & 1.206 & 434.185 \\
\hline Los Rios & 105.692 & 19.851 & 43.035 & & 3 & 7.089 & & 4.421 & 424 & & 1.561 & 182.076 \\
\hline Los Lagos & 14.543 & 17.212 & 24.629 & & & 3.167 & & 567 & 132 & & 281 & 60.531 \\
\hline Aysén & & & 7 & & 23.015 & 3 & & 4.600 & 23 & & 15.179 & 42827 \\
\hline Magallanes & & & & & 1 & & & 24 & & & 257 & 282 \\
\hline TOTAL & 1.457 .224 & 471.738 & 167.899 & 59.093 & 25.089 & 21.751 & 20.698 & 16.676 & 6.278 & 5.180 & 48.464 & 2.300 .090 \\
\hline
\end{tabular}

Muy mayoritariamente las especies más utilizadas son Pinus radiata, Eucalyptus globulus y Eucalyptus nitens, la primera representa el $63 \%$ de la superficie total plantada, la segunda el $21 \%$ y la tercera el $7 \%$, totalizando en conjunto el $91 \%$ de las plantaciones. En la superficie restante participan los arbustos forrajeros (Atriplex spp) con un 2,6\%, Pinus ponderosa, otros eucaliptos y tamarugo (Prosopis tamarugo) con participaciones cercanas al $1 \%$ cada una, y pino oregón (Pseudotsuga menziesii), álamos (Populus spp), algarrobos (Prosopis spp) y otras especies con participaciones menores.

Las Regiones del norte, desde Arica y Parinacota hasta Atacama, son de condiciones desérticas, las precipitaciones son muy escasas o nulas la vegetación natural ocurre sólo en valles y en precordillera y altiplano, o en situaciones localizadas con napas freáticas cercanas a la superficie, como es el caso de la Pampa del Tamarugal en la Región de Tarapacá donde existen formaciones naturales de tamarugo (Prosopis tamarugo) y algarrobo (Prosopis alba) y plantaciones con estas mismas especies.

Las Regiones de Coquimbo a Maule constituyen las zonas áridas y semiáridas, con precipitaciones en torno a $80 \mathrm{~mm}$ anuales en el norte y cercanas a los $800 \mathrm{~mm}$ anuales por el 
sur, muy concentradas en invierno y muy irregulares, tanto en cada año como a través de los años, siendo frecuentes los períodos de sequía en los que, por un año o varios, los montos de precipitación caen muy por debajo de los normales.

En términos generales, la zona árida corresponde a la Región de Coquimbo y la semiárida se extiende de Valparaíso al sur, donde los sectores de Valle Central o secano interior, reciben menores precipitaciones que aquellos de Cordillera de la Costa y Precordillera y Cordillera Andina. A través del Valle Central esta zona semiárida se extiende algo más al sur hasta la Región del Bio Bio.

En la zona árida existe una masa importante de plantaciones de arbustos forrajeros, principalmente Atriplex nummularia, de origen australiano, y Atriplex repanda, nativo de la zona, además de plantaciones con otras especies nativas como algarrobo (Prosopis chilensis) y plantaciones de acacia (Acacia saligna), también de origen australiano, de la cual existen unas 12.000 ha. En áreas de valles, zonas costeras y zonas precordilleranas hay plantaciones de eucaliptos, principalmente Eucalyptus globulus y secundariamente algunos más resistentes a condiciones de escasa pluviometría, como Eucalyptus camaldulensis, Eucalypytus cladocalyx, Eucalyptus sideroxylon y otros.

En la zona semiárida aparecen ya importantes superficies de Pinus radiata y Eucalyptus globulus, principalmente en áreas costeras de 500 mm anuales de precipitación y más. En áreas interiores en tanto, en condiciones más adversas con precipitaciones por debajo de los $500 \mathrm{~mm}$ anuales y un prolongado período seco estival, existen algunas plantaciones de Eucalyptus globulus, Eucalyptus camaldulensis y algunas nativas como quillay (Quillaja saponaria) y algarrobo (Prosopis chilensis).

Esta zona reúne también la mayor concentración de plantaciones comerciales de álamo, principalmente en las Regiones de O'Higgins y del Maule, y de sauces en la Región de O’Higgins. Estas plantaciones están muy localizadas y se ubican muy mayoritariamente en suelos agrícolas bajo riego.

No existen álamos nativos en Chile y respecto de los sauces, sólo una especie es nativa, Salix humboldtiana.

Las plantaciones de álamos se efectúan normalmente con material mejorado genéticamente que es propagado en forma agámica (varas o varetas) y corresponden a clones comerciales, frecuentemente híbridos entre dos especies, y conocidos como cultivares. Los más utilizados en las plantaciones son Populus $x$ canadensis "I-214", Populus deltoides "I63/51 Rolando", Populus x canadensis "I-488" y Populus deltoides "cat 64/51", y aún se emplea también Populus nigra "Chile". Todos introducidos de Europa y Norteamérica. (Barros, 2009).

En cuanto a los sauces, las principales especies de sauces existentes en el país son Salix humboldtiana, Salix babilonica, Salix alba y Salix viminalis, la primera nativa y las restantes introducidas desde Asia, Europa y Norteamérica. La más utilizada en plantaciones es Salix viminalis (Barros, 2009). 
Las Regiones de Bio Bio a Los Lagos corresponden a una zona templada húmeda, con precipitaciones anuales de 1.000 a más de $2.500 \mathrm{~mm}$. Esta zona concentra el $67 \%$ de las plantaciones forestales del país, $70 \%$ aproximadamente de las de Pinus radiata y Eucalyptus globulus, casi la totalidad de las de Eucalyptus nitens y de otros eucaliptos, entre los que se cuentan Eucalyptus regnans, Eucalyptus delegatensis, Eucalyptus viminalis y otras, así como también gran parte de las plantaciones de pino oregón (Pseudotsuga menziesii).

En general, solo con la excepción de algunas áreas de secano interior del norte de la Región del Bio Bio y de sectores de precordillera andina por sobre los 700 - 800 msnm, estas regiones del país constituyen muy buenos sitios para el desarrollo de numerosas y variadas especies forestales.

Las Regiones del extremo austral, Aysén y Magallanes, corresponden a una zona templada fría, con presencia de nieves invernales y precipitaciones muy variables, según ubicación; costa, interior o Patagonia.

En Aysén se encuentra gran parte de las plantaciones de Pinus ponderosa y una importante superficie de pino oregón y de otras especies, entre las cuales predominan Pinus contorta, Pinus sylvestris y Larix spp. En Magallanes, bajo condiciones más extremas dadas por bajas temperaturas y vientos, las plantaciones son muy escasas y están representadas por pino oregón y otras coníferas.

Se emplean más de 40 especies en plantaciones forestales, algunas en forma muy localizada, como el tamarugo, en la Región de Tarapacá; los arbustos forrajeros, el algarrobo y la Acacia saligna, en la Región de Coquimbo; los sauces en la Región de O’Higgins; los álamos en sectores de las Regiones de O’Higgins y del Maule; o el pino ponderosa, en la Región de Aysén; y otras, exóticas y nativas, son empleadas aún en reducidas superficies (Cuadro $\mathrm{N}^{\circ} 2$ ).

A pesar de su escasa proporción en la superficie forestada del país, las especies de uso menos tradicional constituyen una valiosa base para diversificar las plantaciones y consecuentemente la producción de materas primas, también para recuperar e integrar a la producción suelos forestales descubiertos, en especial en zonas cuyas condiciones de sitio resultan marginales para el crecimiento de las especies de uso tradicional.

El Instituto Forestal ha apoyado permanentemente esta evolución a través investigaciones de introducción y prueba de nuevas especies, de su propagación y establecimiento, de su silvicultura y manejo, de su mejoramiento genético y de su utilización.

Estos trabajos se continúan desarrollando, para nuevas especies y para especies ya en uso, en diferentes zonas del país. Hoy las prioridades principales están en las zonas áridas y semiáridas y en las zonas templadas frías, donde existen grandes superficies de terrenos descubiertos, para los cuales aún son necesarias más y mejores alternativas productivas, desde la producción de forraje, energía y madera para usos menores hasta madera aserrada y otros productos de mayor valor, de acuerdo con las posibilidades que los sitios ofrecen en cada caso y considerando también las funciones de carácter social y ambiental que las nuevas plantaciones puedan cumplir. 
El presente trabajo presenta resultados preliminares de la evaluación de tres ensayos de especies y progenies establecidos en la Región de Coquimbo, que consideran a las especies Eucalyptus camaldulensis, Eucalyptus cladocalyx, Eucalyptus sideroxylon, Eucalyptus tereticornis, Eucalyptus diversicolor, Eucalyptus tricarpa y Corymbia maculata (ex Eucalyptus maculata). También de tres ensayos clonales de E. camaldulensis establecidos en las regiones de Coquimbo y Valparaiso, donde se evalúa el desempeño de réplicas vegetativas obtenidas por micropropagación de individuos selectos de esta especie.

Los resultados corresponden a una etapa inicial de desarrollo de los ensayos, los cuales seguirán evaluándose en trabajos posteriores para precisar en forma más efectiva las conclusiones que se puedan aplicar en el campo de la selección y mejoramiento genético de las especies forestales apropiadas para forestación en las zonas semiáridas del país. 


\section{Cuadro $\mathrm{N}^{\circ} 2$}

ESPECIES EN USO EN PLANTACIONES FORESTALES EN CHILE

Acacia saligna

Acacia melanoxylon

Acacia meamsii

Acacia dealbata

Acacia caven

Atriplex repanda

Atriplex nummularia

Castanea sativa

Eucalyptus globulus

Eucalyptus nitens

Eucalyptus regnans

Eucalyptus delegatensis

Eucalyptus camaldulensis

Eucalyptus cladocalyx

Eucalyptus tereticomis

Eucalyptus sideroxylon

Juglans regia

Larix decidua

Nothofagus obliqua

Nothofagus dombeyi

Nothofagus alpina

Nothofagus pumilio

Nothofagus antárctica

Pinus radiata

Pinus contorta

Pinus silvestris

Pinus ponderosa

Pinus pinaster

Populus alba

Populus $x$ canescens

(Populus alba $\times$ Populus tremula)

Populus nigra

Populus simonii

Populus deltoides

Populus $x$ canadensis

(Populus deltoides $x$ Populus nigra)

Prosopis tamanugo

Prosopis chilensis

Prosopis alba

Pseudotsuga menziesii

Quillaja saponaria

Robinia pseudoacacia

Salix alba

Salix viminalis

Salix babilónica

Salix humboldtiana
Blue Leaf Wattle, Acacia Azul

Blackwood, Aromo Australiano

Blackwattle

Silver Wattle, Aromo del Pais

Espino

Sereno

Bluegreen Saltbush

Castaño, Chestnut

Blue Gum

Shining Gum

Mountain Ash

Alpine Ash

River Red Gum

Sugar Gum

Forest Red Gum

Mugga, Red Ironbark

Nogal, Walnut

Larch, Larix

Roble

Coihue, Coigúe

Rauli

Lenga

Nirre, Nire

Monterey Pine

Lodgepole Pine

Scots Pine

Ponderosa Pine

Pino Maritimo

Alamo Blanco

Grey Poplar (hibrido)

Lombardy Poplar, Ålamo negro

Simon Poplar

Eastern Cottonwood

Canadian Poplar (hibrido Euramericana)

Tamarugo

Algarrobo

Algarrobo

Douglas Fir, Pino Oregón

Quillay

Robinia, Acacia Falsa

White Willow, Sauce Blanco

Osier, Basket Willow, Sauce Mimbre

Weeping Willow, Sauce LLorón

Sauce Chileno

\section{Australia \\ Australia \\ Australia \\ Australia \\ Chile \\ Chile \\ Australia \\ Europa \\ Australia \\ Australia \\ Australia \\ Australia \\ Australia \\ Australia \\ Australia \\ Australia \\ Asia \\ Europa \\ Chile \\ Chile \\ Chile \\ Chile \\ Chile}

Norte América

Norte América

Europa y Asia

Norte América

Europa y África

Europa y África

Europa y África; Europa y Asia

Europa y Asia

Asia

Norte América

Norte América; Europa y Asia

Chile

Chile

Chile

Norte América

Chile

Norte América

Europa y Asia

Europa y Asia

Asia

Chile 


\section{OBJETIVOS}

\section{General}

Generar antecedentes para orientar la selección y mejoramiento genético de especies sugeridas para la forestación de zonas áridas y semiáridas, donde el déficit hídrico impuesto por las reducidas e irregulares precipitaciones restringe el cultivo de plantaciones con especies tradicionales.

\section{Específicos}

Evaluar el comportamiento de especies y progenies de Eucalyptus camaldulensis, Eucalyptus cladocalyx, Eucalyptus sideroxylon, Eucalyptus tereticornis, Eucalyptus diversicolor, Eucalyptus tricarpa y Corymbia maculata en tres ensayos establecidos en la Región de Coquimbo.

Evaluar el comportamiento de clones de Eucalyptus camaldulensis en tres ensayos instalados en las Regiones de Coquimbo y Valparaíso.

\section{MATERIAL Y MÉTODO}

\section{Ensayos Especies-Progenies}

Se evalúa la respuesta al sitio de 7 especies en tres ensayos establecidos en la Región de Coquimbo (Cuadro $\mathrm{N}^{\circ} 3$ ).

\section{Cuadro $\mathrm{N}^{\circ} 3$}

\section{UBICACIÓN ENSAYOS ESPECIES-PROGENIES}

\begin{tabular}{|l|c|c|c|}
\hline REGIÓN & PROVINCIA & COMUNA & ENSAYO \\
\hline Coquimbo & Choapa & Canela & Huentelauquén \\
\hline Coquimbo & Choapa & Los Vilos & Caracas \\
\hline Coquimbo & Choapa & Illapel & Tunga Norte \\
\hline
\end{tabular}

Las especies en ensayo y la cantidad de progenies en cada caso son las indicadas en el Cuadro $\mathrm{N}^{\circ} 4$. 


\section{Cuadro $\mathrm{N}^{\circ} 4$}

ESPECIES Y PROGENIES CONSIDERADAS EN CADA ENSAYO

\begin{tabular}{|l|c|c|c|}
\hline \multirow{2}{*}{ Especies } & \multicolumn{3}{|c|}{ Número de Progenies por Ensayo } \\
\cline { 2 - 4 } & Hda. Caracas & Tunga Norte & Huentelauquén \\
\hline E. camaldulensis & 22 & 20 & 22 \\
\hline E. cladocalyx & 18 & 17 & 17 \\
\hline E. sideroxylon & 6 & 40 & 6 \\
\hline E. tereticornis & 4 & & 4 \\
\hline E. diversicolor & & 3 & \\
\hline E. tricarpa & & 50 & \\
\hline Corymbia maculata & & $\mathbf{1 3 5}$ & \\
\hline Total & $\mathbf{5 0}$ & & \\
\hline
\end{tabular}

El ensayo de la Hacienda Caracas fue establecido en el año 2005 en la Comuna de los Vilos, Región de Coquimbo. Se encuentra en terrenos correspondientes a planicies litorales donde predomina un clima de estepa con nubosidad abundante y oscilaciones térmicas bajas debido al efecto moderador del mar. Las precipitaciones, unos $210 \mathrm{~mm}$, se concentran en más de un $80 \%$ en los meses invernales exhibiendo un periodo seco prolongado.

El Ensayo Tunga Norte se estableció el año 2006 en terrenos de la comunidad agrícola del mismo nombre, en la Comuna de Illapel, Región de Coquimbo. El suelo corresponde a un aridsol de origen granítico y de transición entre pardos cálcico a pardos no cálcicos. El clima imperante en el área es del tipo mediterráneo árido, caracterizado por una precipitación invernal promedio de $220 \mathrm{~mm}$ anuales y un largo periodo seco de 8 a 11 meses.

El ensayo Huentelauquén se estableció el año 2006 en la Comuna de Canela, Región de Coquimbo, en una condición de clima mediterráneo árido con nubosidad abundante, precipitación anual cercana a los $200 \mathrm{~mm}$ y periodo seco prolongado.

Los tres ensayos tienen un diseño de bloques completos al azar. El ensayo Caracas consta de 18 bloques, que incluyen 50 progenies en parcelas de un árbol individual; Tunga Norte comprende 4 bloques, con 135 progenies en parcelas lineales de cuatro plantas cada una; y Huentelauquén considera 12 bloques donde hay 49 progenies en parcelas lineales de 3 plantas cada una. En consecuencia, en cada ensayo las progenies están representadas por un total de 18,16 y 36 plantas, respectivamente.

Las progenies en ensayo corresponden a colecciones de origen australiano y se incluye en algunos casos una progenie local procedente de un ensayo anterior en la Región de Coquimbo (Cuz Cuz).

La evaluación de los ensayos consideró una medición efectuada el año 2009, equivalente a tres años de edad para la plantación de Caracas y dos años de edad para Tunga Norte y Huentelauquén. En la ocasión se registró el crecimiento en altura (ALT) y diámetro de cuello (DAC), valores que fueron analizados mediante procedimientos estadísticos descriptivos, de 
análisis de varianza y pruebas de comparaciones múltiples de medias (Duncan, Tuckey y Scott y Knott). .

Como parámetro complementario se analizó el comportamiento de la variable supervivencia (SUP) y se construyó un estimador de la biomasa producida por el árbol medio (E), que integra a las variables altura y diámetro, ponderadas por la supervivencia, y expresado en $\mathrm{cm}^{3}$.

$$
\mathrm{E}\left(\mathrm{cm}^{3}\right)=\operatorname{ALT}(\mathrm{cm}) \times \operatorname{DAC}^{2}\left(\mathrm{~cm}^{2}\right) \times \operatorname{SUP}(\%)
$$

La determinación de las medias de supervivencia consideró todas las plantas vivas, independiente de su estado, mientras que para determinar las medias de altura, las bases de datos fueron depuradas usando la información de estado, para eliminar del promedio a las plantas dañadas.

Respecto de los resultados que están mostrando las progenies de cada especie por separado, dentro del marco de la investigación sobre mejoramiento genético que desarrolla INFOR, están siendo procesados en forma más detallada en un análisis procedenciaprogenie, conducente a desarrollar estrategias de mejoramiento genético para las especies más destacadas.

No obstante, se presentan aquí resultados resumidos, en los que se indica la progenie con el mejor y el peor resultado para cada especie y para el total del ensayo, como una referencia que permite visualizar el potencial que representa la selección sucesiva especie-procedenciaprogenie y mejoramiento genético a partir de material superior en cada caso. Para este fin se usa el estimador $\mathrm{E}$ descrito anteriormente.

\section{Ensayos Clones Eucalyptus camaldulensis}

Se evalúa la respuesta al sitio de 13 clones de E. camaldulensis establecidos en tres ensayos clonales en las Regiones de Coquimbo y Valparaíso (Cuadro $N^{\circ} 5$ ). Estos ensayos fueron plantados el año 2005 y se evalúa la medición correspondiente a los primeros 10 meses de crecimiento en terreno.

Los clones considerados son réplicas vegetativas de árboles seleccionados en función de la superioridad de su desempeño en plantaciones experimentales establecidas por INFOR a comienzos de la década de los '90 en Tantehue (Melipilla, Región Metropolitana) y en Longotoma (La Ligua, Región de Valparaíso). 


\section{Cuadro $\mathrm{N}^{\circ} 5$ UBICACIÖN ENSAYOS CLONES Eucalyptus camaldulensis}

\begin{tabular}{|l|c|c|c|}
\hline REGIÓN & PROVINCIA & COMUNA & ENSAYO \\
\hline Coquimbo & Elqui & Tongoy & El Tangue \\
\hline Coquimbo & Choapa & Illapel & Cuz Cuz \\
\hline Valparaíso & Petorca & La Ligua & Pullally \\
\hline
\end{tabular}

El ensayo El Tangue se encuentra en un clima clasificado como de estepa con nubosidad abundante. Se caracteriza por abundante nubosidad baja. La cercanía del mar produce amplitudes térmicas bajas. Las precipitaciones presentan un régimen frontal, con máximos en el invierno (junio, julio y agosto) donde precipita cerca del $80 \%$ del total anual. En El Tangue caen $107 \mathrm{~mm}$ anuales.

El ensayo Cuz-Cuz se ubica en una situación de clima de estepa cálido, que se caracteriza por la sequedad del aire y la ausencia de nubosidad. Sus temperaturas son mayores que en la costa, las precipitaciones son muy irregulares y escasas, con una media anual de unos $210 \mathrm{~mm}$, y los períodos de sequía son característicamente extensos (8 meses o más). El sitio corresponde a la parte baja de una ladera orientada hacia al sur-este, con una acentuada pendiente de cerca del $90 \%$ y suelo severamente compactado.

El ensayo Pullally corresponde a una zona mediterránea con marcada influencia marina. La precipitación media anual es de $230 \mathrm{~mm}$ fuertemente concentrada en los meses invernales. $\mathrm{La}$ influencia costera se manifiesta en temperaturas templadas que no sobrepasan los $25^{\circ} \mathrm{C}$, con una muy baja incidencia de heladas. El suelo corresponde a arenas originadas en una duna fósil de baja fertilidad (Smith, 1997).

Cada ensayo se compone de aproximadamente 13 clones (Cuadro $N^{\circ} 6$ ), distribuidos en 4 bloques al azar. En cada uno de los bloques, cada clon se encuentra representado por una parcela lineal de 4 rametos, a un espaciamiento de $3 \times 3 \mathrm{~m}$. No obstante, con el fin de aprovechar el material genético y conformar bloques cuadrados, se incorporaron parcelas adicionales con aquellos clones que presentaban más rametos, estos también se utilizaron para reemplazar a aquellos clones que por su baja disponibilidad de plantas no alcanzaban a ser representados en todos los bloques. Consecuentemente, los diseños definitivos tiene un cierto grado de desbalance y no todos los clones se representan con el mismo número de plantas en cada ensayo. 


\section{Cuadro $\mathrm{N}^{\circ} 6$ \\ IDENTIFICACIÓN DE LOS CLONES DE Eucalyptus camaldulensis INCLUIDOS EN CADA ENSAYO}

\begin{tabular}{|c|c|c|c|c|}
\hline Código Clon & Identificación del Ortet & Pullally & Cuz Cuz & El Tangue \\
\hline 1 & Tantehue Bq 1; proced 2, Prog 16; Arbol 3 & $x$ & $x$ & $x$ \\
\hline 3 & Tantehue Bq 1; proced 9, Prog 98; Arbol 3 & $\mathrm{x}$ & $\mathrm{x}$ & $x$ \\
\hline 5 & Tantehue Bq 2; proced 2, Prog 9; Arbol 1 & $\mathrm{x}$ & $x$ & $x$ \\
\hline 6 & Tantehue Bq 2; proced 8, Prog 87; Arbol 3 & & $x$ & \\
\hline 9 & Tantehue Bq 3; proced 6, Prog 63; Arbol 1 & $\mathrm{x}$ & $x$ & $x$ \\
\hline 11 & Tantehue Bq 4; proced 6, Prog 60; Arbol 1 & $x$ & $x$ & $\mathrm{x}$ \\
\hline 21 & Tantehue Bq 8; proced 3, Prog 22; Arbol 4 & & $x$ & \\
\hline 27 & Tantehue Bq 9; proced 2, Prog 19; Arbol 2 & & $x$ & $x$ \\
\hline 29 & Tantehue Bq 10; proced 5, Prog 52; Arbol 1 & $\mathrm{x}$ & $x$ & $x$ \\
\hline 31 & Tantehue Bq 10; proced 8, Prog 81; Arbol 4 & $x$ & $x$ & $x$ \\
\hline 43 & Longotoma $32^{\circ} 24,665^{\prime} ; 71^{\circ} 21,724^{\prime}$ & $x$ & $x$ & $x$ \\
\hline 44 & Longotoma $32^{\circ} 24,676^{\prime} ; 71^{\circ} 20,670^{\prime}$ & & $x$ & $x$ \\
\hline 50 & Tantehue Plantación masiva & $x$ & $x$ & $x$ \\
\hline
\end{tabular}

Al igual que en los ensayos de especies y progenies, las mediciones registraron el crecimiento en altura y diámetro de cuello. Como parámetro complementario se incluye también el comportamiento de la variable supervivencia. La determinación de las medias de supervivencia consideró todas las plantas vivas, independiente de su estado, mientras que para determinar las medias de altura, las bases de datos fueron depuradas usando la información de estado, para eliminar del promedio a las plantas dañadas.

\section{RESULTADOS Y DISCUSIÓN}

\section{Ensayos Especies y Progenies}

El análisis de las especies en los diferentes ensayos permite apreciar un mejor desempeño general de las plantas en el ensayo Caracas, con valores de supervivencia que resultan claramente superiores. También son superiores los valores de altura y diámetro de cuello, pero en este caso las diferencias se confunden con la diferencia de edad de los ensayos;

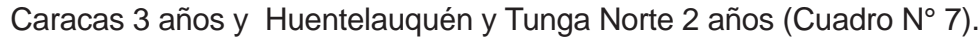

El mejor resultado en este ensayo obedece muy probablemente a las condiciones ambientales más favorables, particularmente la cercanía al mar y el efecto de neblinas costeras, lo que permite que las tres especies puedan manifestar un comportamiento muy similar. En los ensayos restantes, las condiciones más adversas limitan el desarrollo de las especie menos adaptadas a condicione de aridez, haciendo manifiesta la diferencias entre ellas.

En general se observa que en las condiciones más severas de Huentelauquén y Tunga Norte, Eucalyptus camaldulensis y E. cladocalyx continúan manifestando los mejores 
crecimientos, mientras que E. sideroxylon, E. tereticornis, E. tricarpa y E. diversicolor presentan valores menores. También se destaca el buen desarrollo de Corymbia maculata, que resulta similar al de las especies de mejor desempeño.

\section{Cuadro $\mathrm{N}^{\circ} 7$ \\ DESEMPEÑO LAS DISTINTAS ESPECIES FORESTALES SEGÚN ENSAYOS}

\begin{tabular}{|c|c|c|c|c|c|c|c|c|c|}
\hline \multirow{3}{*}{ Especies } & \multicolumn{9}{|c|}{ ENSAYOS } \\
\hline & \multicolumn{3}{|c|}{ Caracas (3 años) } & \multicolumn{3}{|c|}{ Huentelauquén (2 años) } & \multicolumn{3}{|c|}{ Tunga Norte (2 años) } \\
\hline & $\begin{array}{r}\text { SUP } \\
\text { (\%) }\end{array}$ & $\begin{array}{l}\text { ALT } \\
\text { (cm) }\end{array}$ & $\begin{array}{l}\text { DAC } \\
(\mathrm{mm})\end{array}$ & $\begin{array}{l}\text { SUP } \\
(\%)\end{array}$ & $\begin{array}{l}\text { ALT } \\
(\mathrm{cm})\end{array}$ & $\begin{array}{c}\text { DAC } \\
(\mathrm{mm})\end{array}$ & $\begin{array}{l}\text { SUP } \\
\text { (\%) }\end{array}$ & $\begin{array}{l}\text { ALT } \\
(\mathrm{cm})\end{array}$ & $\begin{array}{l}\text { DAC } \\
(\mathrm{mm})\end{array}$ \\
\hline Eucalyptus camaldulensis & 84,6 & $234,3^{a}$ & $43,9^{a}$ & 65,2 & $98,6^{a}$ & $14,1^{b}$ & 66,9 & $118,2^{a}$ & $22,5^{b}$ \\
\hline Eucalyptus cladocalyx & 87,7 & $243,3^{a}$ & $44,6^{a}$ & 51,4 & $97,8^{\mathrm{a}}$ & $17,8^{\mathrm{a}}$ & 22,8 & $117,2^{\mathrm{a}}$ & $27,8^{\mathrm{a}}$ \\
\hline Eucalyptus sideroxylon & 91,7 & $239,3^{a}$ & $44,8^{a}$ & 44,3 & $72,1^{\mathrm{b}}$ & $11,6^{\mathrm{c}}$ & 45,0 & $80,1^{b}$ & $13,7^{\mathrm{d}}$ \\
\hline Eucalyptus tereticornis & 81,9 & $238,9^{a}$ & $43,8^{a}$ & 52,0 & $72,4^{\mathrm{b}}$ & $11,7^{\mathrm{c}}$ & & & \\
\hline Eucalyptus diversicolor & & & & & & & 60,4 & $96,3^{\mathrm{a}}$ & $20,7^{b}$ \\
\hline Eucalyptus tricarpa & & & & & & & 45,3 & $106,2^{\mathrm{a}}$ & $17,8^{\mathrm{c}}$ \\
\hline Corymbia maculata & & & & & & & 41,3 & $117,4^{\mathrm{a}}$ & $21,2^{b}$ \\
\hline Promedio & 86,3 & 239,2 & 44,4 & 56,8 & 94,3 & 15,0 & 45,7 & 102,0 & 18,5 \\
\hline
\end{tabular}

Letras distintas en una misma columna indican diferencias significativas (Scott y Knott para $\alpha=0,05$ )

\section{- Ensayo Caracas}

Las cuatro especies evaluadas en el ensayo Caracas a la edad de tres años presentan un comportamiento muy similar. En efecto, el análisis de varianza indica que existen diferencias estadísticamente significativas entre progenies dentro de las especies, pero que entre las especies esta diferencia no existe, de modo que las cu atro forman un mismo grupo homogéneo de acuerdo a los resultados de tres pruebas de comparaciones múltiples de medias (Duncan, Tuckey y Scott y Knott).

Los valores medios del ensayo en su conjunto para las variables analizadas fueron $86,3 \%$ de supervivencia, $239,2 \mathrm{~cm}$ de altura y $44,4 \mathrm{~mm}$ de diámetro de cuello. Una visión gráfica del comportamiento de las especies se presenta en la Figura $N^{\circ} 1$, la cual da cuenta del similar comportamiento de las cuatro especies evaluadas. 


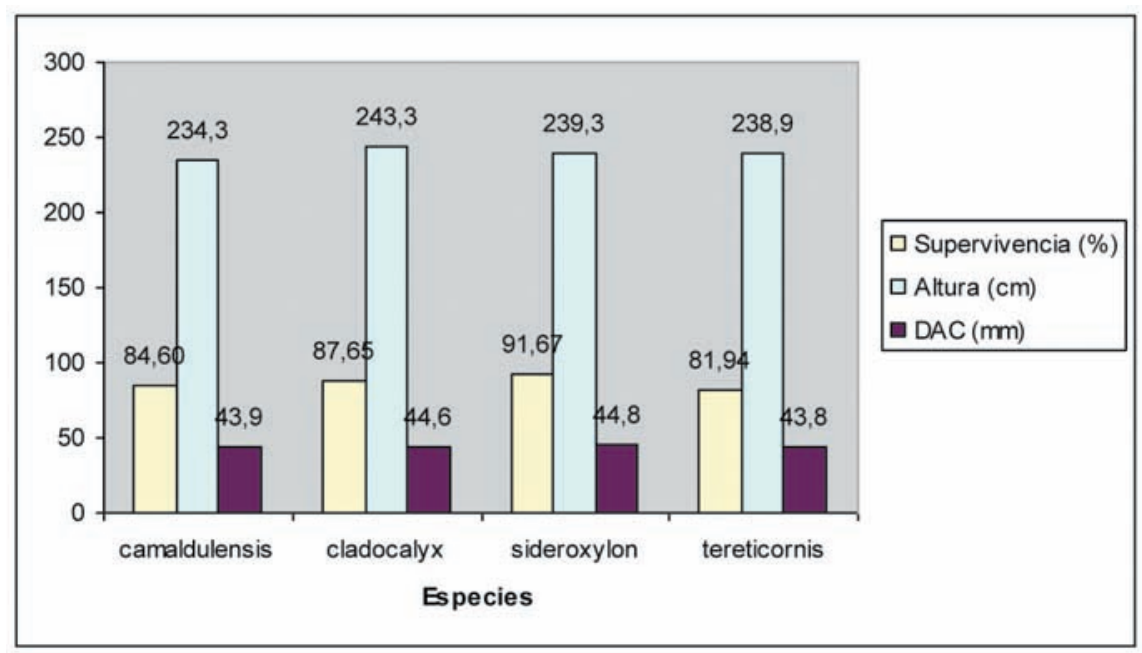

\section{Figura $N^{\circ} 1$ \\ VALORES MEDIOS DE SUPERVIVENCIA, ALTURA Y DIÁMETRO DE CUELLO POR ESPECIE EDAD DE TRES AÑOS - ENSAYO CARACAS}

Los valores observados resultan de una magnitud equivalente a los obtenidos anteriormente en ensayos de progenies de Eucalyptus camaldulensis. En efecto, para cuatro ensayos de esta especie, evaluados a la edad de 42 a 44 meses, Alvear y Gutiérrez (1995) señalan los valores de altura, DAC y supervivencia que se muestran en el Cuadro $\mathrm{N}^{\circ} 8$.

\section{Cuadro $\mathrm{N}^{\circ} 8$}

PARAMETROS MEDIOS EN CUATRO ENSAYOS DE PROGENIES DE Eucalyptus camaldulensis - EVALUADOS A LA EDAD DE 42 a 44 MESES

\begin{tabular}{|l|c|c|c|c|c|c|}
\hline REGIÓN & PROVINCIA & COMUNA & ENSAYO & $\begin{array}{c}\text { SUP } \\
(\%)\end{array}$ & $\begin{array}{c}\text { ALT } \\
(\mathbf{c m})\end{array}$ & $\begin{array}{c}\text { DAC } \\
(\mathbf{m m})\end{array}$ \\
\hline Coquimbo & Petorca & La Ligua & Longotoma & 93,2 & 328 & 52,4 \\
\hline Valparaiso & Valparaiso & Casablanca & Mel Mel & 83,3 & 338 & 58,1 \\
\hline Metropolitana & Melipilla & Melipilla & Tantehue & 96,9 & 659 & \\
\hline O'Higgins & Colchagua & & La Paila & 78,1 & 257 & \\
\hline
\end{tabular}

Las variables de crecimiento, son mayores en los ensayos reportados por estos autores respecto de lo observado en Caracas, diferencias que obedecen por una parte a la diferencia de edad de los ensayos comparados y, en el caso del ensayo Tantehue, a condiciones más favorables del sitio y al origen selecto de las procedencias consideradas en su establecimiento.

Respecto de los resultados que están mostrando las progenies de cada especie por separado, los resultados resumidos, en los que se indica la progenie con el mejor y el peor 
resultado para cada especie y para el total del ensayo, según el estimador de biomasa (E), son los indicados en el (Cuadro $\mathrm{N}^{\circ}$ 9).

\section{Cuadro $\mathrm{N}^{\circ} 9$ \\ PROGENIE CON EL MAYOR Y EL MENOR RESULTADO POR ESPECIE SEGÚN ESTIMADOR DE BIOMASA (E) EDAD TRES AÑOS - ENSAYO CARACAS}

\begin{tabular}{|c|c|c|c|c|c|c|}
\hline \multirow{2}{*}{ ESPECIES } & \multicolumn{2}{|c|}{ PROGENIES } & \multirow{2}{*}{$\begin{array}{l}\text { SUP } \\
\text { (\%) }\end{array}$} & \multirow{2}{*}{$\begin{array}{l}\text { ALT } \\
(\mathrm{cm})\end{array}$} & \multirow{2}{*}{$\begin{array}{l}\text { DAC } \\
(\mathrm{mm})\end{array}$} & \multirow{2}{*}{$\underset{\left(\mathrm{cm}^{3}\right)}{E}$} \\
\hline & $\left(N^{\circ}\right)$ & Código & & & & \\
\hline \multirow{2}{*}{ Eucalyptus camaidulensis } & \multirow{2}{*}{22} & 51024 & 83,3 & 275,9 & 50,2 & 5792 \\
\hline & & 41040 & 88,9 & 193,3 & 33,9 & 1975 \\
\hline \multirow{2}{*}{ Eucalyptus cladocalyx } & \multirow{2}{*}{18} & 52003 & 94,4 & 271,6 & 47,8 & 5858 \\
\hline & & A 25 & 88,9 & 216,4 & 41,4 & 3297 \\
\hline \multirow{2}{*}{ Eucalyptus sideroxylon } & \multirow{2}{*}{6} & 53004 & 94,4 & 257,8 & 49,2 & 5891 \\
\hline & & 53002 & 88,9 & 206,1 & 41,7 & 3186 \\
\hline \multirow{2}{*}{ Eucalyptus tereticornis } & \multirow{2}{*}{4} & 53002 & 94,4 & 234,3 & 48,5 & 5203 \\
\hline & & 450 sp1 & 77,8 & 237,1 & 37,6 & 2608 \\
\hline Todas & & & & & & $\begin{array}{l}5891 \\
1975\end{array}$ \\
\hline
\end{tabular}

$E=\operatorname{SUP}(\%) \times A L T(\mathrm{~cm}) \times D^{2} C^{2}(\mathrm{~cm})$

Las progenies de mejor desempeño, duplican y hasta triplican en el valor del estimador $E$, a las de menor resultado. En este ensayo, la progenie con menor estimador de biomasa ( $E$. camaldulensis 41040) es triplicada por la que obtiene el valor más alto (E. sideroxylon 53004).

La variación entre progenies de E. camaldulensis es coherente con la observada en otros ensayos realizados en zonas áridas y semiáridas, que también exhiben gran variabilidad entre familias y procedencias. En este caso, el valor medio de E para las 22 progenies evaluadas en el ensayo es de $3820 \mathrm{~cm}^{3}$, la progenie 51024 registra $5792 \mathrm{~cm}^{3}$ y la progenie 41040 sólo 1975 $\mathrm{cm}^{3}$.

Se destaca y confirma la importancia de la selección de especies, pero más aún de la posterior selección genética, fácil resulta prever las diferencias que se podrían producir en el desarrollo de plantaciones de Eucalyptus camaldulensis, en el mismo sitio o uno equivalente, originadas en semillas de de la progenie 41040 ó en clones obtenidos de árboles plus de la progenie 51024.

\section{- Ensayo Huentelauquén}

En este ensayo se consideran las mismas especies que en el de Caracas, pero en este caso se observa un comportamiento distinto de las especies ensayadas (Figura $\mathrm{N}^{\circ} 2$ ). 


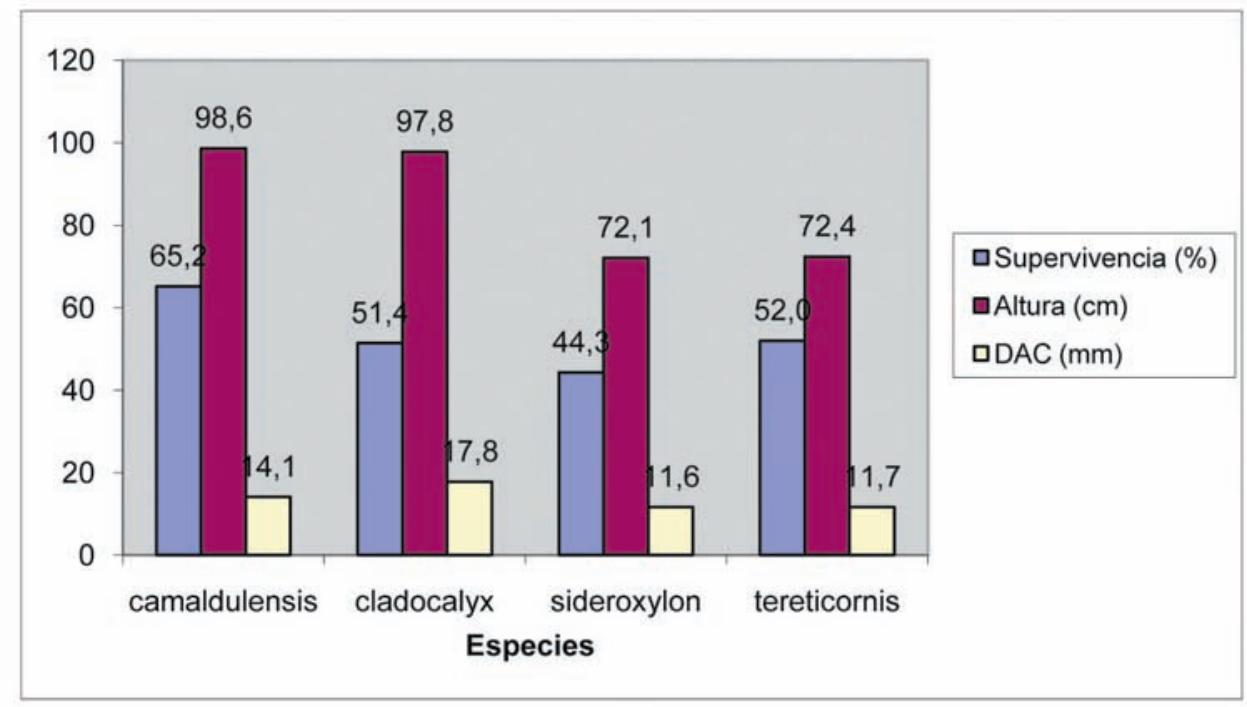

Figura $N^{\circ} 2$

\section{VALORES MEDIOS DE SUPERVIVENCIA, ALTURA Y DIÁMETRO DE CUELLO POR ESPECIE EDAD DE DOS AÑOS - ENSAYO HUENTELAUQUÉN}

El análisis de varianza y prueba de comparaciones múltiples de medias (Scott y Knott, $\alpha=0,05)$ indican que para la altura existen dos grupos con un desempeño estadísticamente diferente. Eucalyptus tereticornis y E. sideroxylon conforman un grupo con altura significativamente menor a la del grupo compuesto por Eucalyptus camaldulensis y $E$. cladocalyx. En el caso del diámetro de cuello se observa una situación similar; Eucalyptus cladocalyx y E. sideroxylon forman el grupo de menor valor, Eucalyptus camaldulensis un valor intermedio y Eucalyptus cladocalyx el mayor de las cuatro especies evaluadas (Cuadro $\mathrm{N}^{\circ} 7$ ).

La situación anterior confirma antecedentes previos respecto al adecuado desarrollo de Eucalyptus camaldulensis y E. cladocalyx respecto a otras especies alternativas para forestar en zonas áridas.

En cuanto a las progenies, se mantiene la tendencia dada por las especies, y las diferencias entre la mayor y menor progenie son muy marcadas. Los valores de $\mathrm{E}$ son considerablemente más bajos que en el ensayo anterior y las diferencias proporcionalmente más grandes debido principalmente a la menor edad. 


\section{Cuadro $\mathrm{N}^{\circ} 10$}

PROGENIE CON EL MAYOR Y EL MENOR RESULTADO POR ESPECIE SEGÚN ESTIMADOR DE BIOMASA (E) EDAD DOS AÑOS - ENSAYO HUENTELAUQUÉN

\begin{tabular}{|c|c|c|c|c|c|c|}
\hline \multirow{2}{*}{ ESPECIES } & \multicolumn{2}{|c|}{ PROGENIES } & \multirow{2}{*}{$\begin{array}{l}\text { SUP } \\
(\%)\end{array}$} & \multirow{2}{*}{$\begin{array}{l}\text { ALT } \\
(\mathrm{cm})\end{array}$} & \multirow{2}{*}{$\begin{array}{c}\text { DAC } \\
(\mathrm{mm})\end{array}$} & \multirow{2}{*}{$\begin{array}{c}\mathrm{E} \\
\left(\mathrm{cm}^{3}\right)\end{array}$} \\
\hline & $\left(N^{*}\right)$ & Código & & & & \\
\hline \multirow{2}{*}{ Eucalyptus camaldulensis } & \multirow{2}{*}{22} & 51032 & 75,0 & 137,5 & 19,9 & 408 \\
\hline & & 131009A & 61,9 & 68,4 & 10,8 & 49 \\
\hline \multirow{2}{*}{ Eucalyptus cladocalyx } & \multirow{2}{*}{17} & 42011 & 64,2 & 122,0 & 22,6 & 400 \\
\hline & & 42017 & 50,0 & 74,3 & 12,9 & 62 \\
\hline \multirow{2}{*}{ Eucalyptus sideroxylon } & \multirow{2}{*}{6} & 53002 & 53,8 & 87,6 & 15,8 & 118 \\
\hline & & 53003 & 48,5 & 53,2 & 7,0 & 13 \\
\hline \multirow{2}{*}{ Eucalyptus tereticornis } & \multirow{2}{*}{4} & $450 S P 1$ & 60,6 & 75,8 & 12,2 & 68 \\
\hline & & $450 \mathrm{SP} 4$ & 58,3 & 52,8 & 8,0 & 20 \\
\hline Todas & & & & & & $\begin{array}{r}408 \\
13\end{array}$ \\
\hline
\end{tabular}

\section{- Ensayo Tunga Norte}

También evidencia un distinto comportamiento de las especies ensayadas, destacándose al igual que en las unidades anteriores, el desempeño alcanzado por Eucalyptus camaldulensis y E. cladocalyx, los cuales junto a Corymbia maculata alcanzan los mejores valores de altura y diámetro de cuello. Por el contrario, Eucalyptus sideroxylon vuelve a presentar los valores más bajos en ambas variables, tal como se observó en el ensayo Huentelauquén (Figura $N^{\circ} 3$ ).

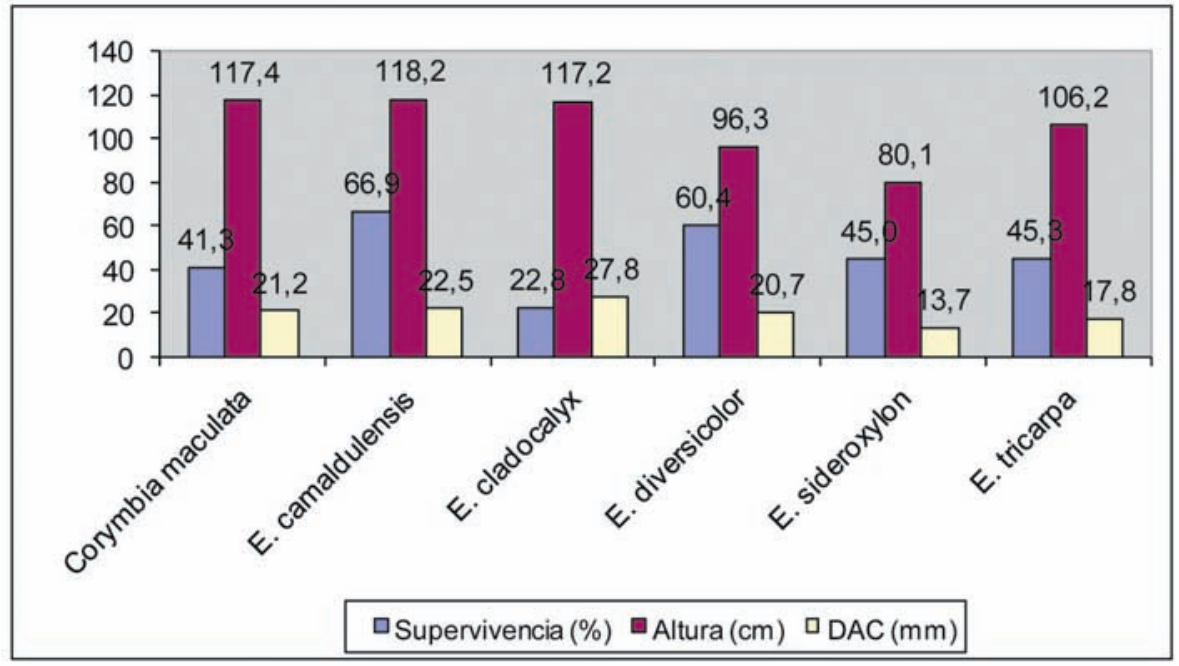

Figura $\mathrm{N}^{\circ} 3$

VALORES MEDIOS DE SUPERVIVENCIA, ALTURA Y DIÁMETRO DE CUELLO POR ESPECIE EDAD DOS AÑOS - ENSAYO TUNGA NORTE 
Los resultados de las progenies en tanto, muestran igual tendencia respecto del comportamiento de las especies, destaca notoriamente la mejor progenie de Eucalyptus camaldulensis respecto de todas las demás progenies del ensayo de esta y las otras especies, y se repiten las marcadas diferencias entre la mayor y menor progenie en cada especie.

Eucalyptus tricarpa y Corymbia maculata, son especies no probadas anteriormente en el país y no incluidas en los dos ensayos anteriores. Eucalyptus tricarpa es originario del sur de Australia, zonas costeras de New South Wales y Victoria. Corymbia maculata proviene de estos mismos estados y de Queensland. Anteriormente se las conocía como Eucalyptus sideroxylon ssp tricarpa, y como Eucalyptus maculata. Sus resultados son interesantes, presentan una buena altura y, pese a que son originarias de áreas algo más húmedas, muestran supervivencias comparables con las de las otras especies en el ensayo.

Eucalyptus diversicolor en tanto, fue probado en anteriores ensayos de INFOR en los '60s y '70s, exhibiendo variables resultados. Su zona de origen es el extremo sur de Western Australia, en áreas de mayores precipitaciones que las del ensayo, es un árbol de gran tamaño, los australianos los señalan como una de las dos latifoliadas más altas en el mundo, junto con Eucalypus regnans. Sus resultados hasta ahora resultan de interés, por cuanto su altura y supervivencia es comparable a la de las otras especies.

\section{Cuadro $\mathrm{N}^{\circ} 11$}

PROGENIE CON EL MAYOR Y EL MENOR RESULTADO POR ESPECIE SEGÚN ESTIMADOR DE BIOMASA (E) EDAD DOS AÑOS - ENSAYO TUNGA NORTE

\begin{tabular}{|c|c|c|c|c|c|c|}
\hline \multirow{2}{*}{ ESPECIES } & \multicolumn{2}{|c|}{ PROGENIES } & \multirow{2}{*}{$\begin{array}{l}\text { SUP } \\
\text { (\%) }\end{array}$} & \multirow{2}{*}{$\begin{array}{l}\text { ALT } \\
(\mathrm{cm})\end{array}$} & \multirow{2}{*}{$\begin{array}{c}\text { DAC } \\
(\mathrm{mm})\end{array}$} & \multirow{2}{*}{$\begin{array}{c}\mathrm{E} \\
\left(\mathrm{cm}^{3}\right)\end{array}$} \\
\hline & $\left(\mathrm{N}^{\circ}\right)$ & Código & & & & \\
\hline \multirow{2}{*}{ Eucalyptus camaldulensis } & \multirow{2}{*}{20} & $131008 \mathrm{~A}$ & 58,3 & 204,3 & 42,3 & 2131 \\
\hline & & 41042 & 43,8 & 82,9 & 15,1 & 83 \\
\hline \multirow{2}{*}{ Eucalyptus cladocalyx } & \multirow{2}{*}{17} & 52003 & 43,8 & 149,3 & 37,1 & 900 \\
\hline & & 42005 & 0,0 & & & 1 \\
\hline \multirow{2}{*}{ Eucalyptus sideroxylon } & \multirow{2}{*}{40} & S5-19 & 81,3 & 91,2 & 17,8 & 235 \\
\hline & & 53003 & 6,3 & 45,0 & 11,0 & d \\
\hline \multirow{2}{*}{ Eucalyptus diversicolor } & \multirow{2}{*}{3} & $450 \mathrm{SP} 3$ & 62,5 & 106,6 & 23,3 & 362 \\
\hline & & $450 \mathrm{SP} 2$ & 62,5 & 79,5 & 17,9 & 159 \\
\hline \multirow{2}{*}{ Eucalyptus tricarpa } & \multirow{2}{*}{50} & T11-44 & 62,5 & 156,2 & 24,8 & 600 \\
\hline & & T8-27 & 6,3 & 35,0 & 5,0 & 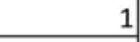 \\
\hline \multirow{2}{*}{ Corimbia maculata } & \multirow{2}{*}{5} & M1-2 & 33,3 & 122,5 & 28,8 & 338 \\
\hline & & M1-4 & 35,0 & 121,1 & 20,0 & 170 \\
\hline Todas & & & & & & 2131 \\
\hline
\end{tabular}

Indudablemente estos tres ensayos requieren de evaluaciones de seguimiento por un período más prolongado, de al menos 10 años, muy en especial en lo que se refiere a las progenies. El potencial de mejoramiento es importante, en cada ensayo hay progenies muy destacadas, que superan hasta ahora ampliamente a las de resultados más bajos. 
No obstante la respuesta a los sitios es algo errática, no se repiten las mismas progenies en los primeros lugares de los tres ensayos,.. Como ejemplo, E. camaldulensis progenie 131008A se ubica en primer lugar en Tunga Norte, séptimo lugar en Caracas y octavo lugar en Huentelauquén; la progenie 51024 de la misma especie en primer lugar en Caracas, séptimo en Huentelauquén y dieciocho en Tunga Norte; la progenie 41040 en último lugar en Caracas, sexto lugar en Huentelauquén y segundo lugar en Tunga Norte. Otras como las progenies 131009A y 41042 de esta especie se ubican muy abajo en los tres lugares de ensayo.

La diferencia de sitio entre los tres ensayos no tiene una amplitud tal que permita prever estas diferencias, además el gradiente de sitio que podría asignarse a estos tres sitios, por cercanía a la costa, principalmente tampoco explica los resultados de las progenies en un lugar y otro.

\section{- Ensayos Clones Eucalyptus camaldulensis}

A diferencia de los ensayos de especies y progenies, los de clones de Eucalyptus camaldulensis presentan marcadas diferencias de sitio, dadas principalmente por su distribución latitudinal, y esto se refleja en los resultados.

El ensayo El Tangue ( $30^{\circ} 18^{\prime}$ LS), en la zona costera norte de la Región de Coquimbo, tiene precipitaciones medias de algo más de $100 \mathrm{~mm}$ anuales; el ensayo Cuz Cuz (31 $30^{\circ}$ LS), en la zona interior sur de la misma región, tiene precipitaciones medias en torno a los $210 \mathrm{~mm}$ anuales; en tanto que el ensayo Pullaly ( $\left.32^{\circ} 26^{\prime} \mathrm{LS}\right)$, en la zona costera norte de la Región de Valparaíso, registra precipitaciones medas de $230 \mathrm{~mm}$ anuales. Este último ensayo se encuentra sobe suelos arenosos de antiguas dunas.

Los resultados medios de supervivencia de plantas en los ensayos responden a la latitud, en consecuencia a la precipitación media anual en este caso, y son en el orden anterior 36,53 y $91 \%$, con una media general de $54 \%$.

Los parámetros de altura, diámetro y estimador de biomasa también muestran los más bajos niveles en El Tangue, pero son superiores en Cuz Cuz que en Pullaly, muy probablemente a causa de los suelos arenosos con menor capacidad de retención de humedad de Pullaly. En el Cuadro $\mathrm{N}^{\circ} 12$ se muestra los resultados a 10 meses de edad para el conjunto de los ensayos. 
Cuadro $\mathrm{N}^{\circ} 12$

PARAMETROS MEDIOS EN TRES ENSAYOS DE CLONES DE Eucalyptus camaldulensis EVALUADOS A LA EDAD DE 10 MESES

\begin{tabular}{|c|c|c|c|c|c|c|}
\hline Codigo Clon & $\begin{array}{c}\text { Plantas } \\
\text { Establecidas }\end{array}$ & $\begin{array}{c}\text { Plantas } \\
\text { Vivas }\end{array}$ & $\begin{array}{c}\text { SUP } \\
(\%)\end{array}$ & $\begin{array}{c}\text { ALT } \\
(\mathbf{c m})\end{array}$ & $\begin{array}{c}\text { DAC } \\
(\mathbf{m m})\end{array}$ & $\begin{array}{c}\text { E } \\
\left(\mathrm{cm}^{3}\right)\end{array}$ \\
\hline
\end{tabular}

\begin{tabular}{|c|c|c|c|c|c|c|}
\hline \multicolumn{7}{|l|}{ Tres Ensayos } \\
\hline 9 Tantehue & 39 & 28 & 71,8 & 160 & 17,1 & 336 \\
\hline 31 Tantehue & 86 & 50 & 58,1 & 140 & 14,5 & 171 \\
\hline 29 Tantehue & 56 & 32 & 57,1 & 120 & 14,8 & 150 \\
\hline 1 Tantehue & 100 & 63 & 63,0 & 120 & 13,7 & 142 \\
\hline 3 Tantehue & 83 & 50 & 60,2 & 130 & 13,2 & 136 \\
\hline Promedio* & 692 & 374 & 54,0 & 120 & 13,9 & 125 \\
\hline 43 Longotoma & 100 & 56 & 56,0 & 100 & 13,8 & 107 \\
\hline 50 Tantehue & 60 & 30 & 50,0 & 120 & 12,5 & 94 \\
\hline 5 Tantehue & 46 & 21 & 45,7 & 110 & 12,8 & 82 \\
\hline 11 Tantehue & 56 & 26 & 46,4 & 110 & 12,4 & 78 \\
\hline
\end{tabular}

\begin{tabular}{|l|c|c|c|c|c|r|}
\hline Dos Ensayos (Cuz Cuz y El Tangue) \\
\hline 27 Tantehue & 22 & 3 & 13,6 & 110 & 13,7 & 28 \\
44 Longotoma & 16 & 2 & 12,5 & 80 & 12,5 & 16 \\
\hline
\end{tabular}

\begin{tabular}{|c|c|c|c|c|c|r|}
\hline \multicolumn{1}{|l|}{ Un Ensayo (Cuz Cuz) } \\
\hline 6 Tantehue & 16 & 8 & 50,0 & 140 & 15,8 & 175 \\
21 Tantehue & 12 & 5 & 41,7 & 140 & 15,8 & 146 \\
\hline
\end{tabular}

("Contempla todas las plantas del ensayo, la supervivencia corresponde al total de plantas vivas, en tanto que la determinación de los parámetros medios ha excluido las plantas con daños).

Es prematuro adelantar conclusiones sobre el comportamiento de los clones en estos ensayos con sólo 10 meses de edad. En el conjunto de ensayos y para aquellos clones que están presentes en todos ellos, los resultados medios están fuertemente afectados por los bajos niveles que alcanzan los parámetros medios en El Tangue, no obstante los clones 9, 31, 29, 1 y 3 aparecen por sobre el promedio de acuerdo al estimador de biomasa. El clon 31 está sobre el promedio en los tres ensayos y los clones 9, 43 y 3 están sobre el promedio en dos ensayos.

Si se considera sólo Cuz Cuz y Pullaly, destacan hasta ahora los clones 9, 31 y 43, por sobre el promedio en ambos ensayos y, en especial, el clon 9 que encabeza los resultados hasta ahora en ambos casos, con una supervivencia media de más de $80 \%$, una altura media de $165 \mathrm{~cm}$ y un diámetro medio de $17,8 \mathrm{~mm}$.

En los Cuadros $\mathrm{N}^{\circ} 13, \mathrm{~N}^{\circ} 14$ y $\mathrm{N}^{\circ} 15$ se indica los resultados para cada ensayo separadamente. 
Cuadro $\mathrm{N}^{\circ} 13$

PARAMETROS MEDIOS EN ENSAYO DE CLONES DE Eucalyptus camaldulensis CUZ CUZ - EVALUADO A LA EDAD DE 10 MESES

\begin{tabular}{|c|c|c|c|c|c|c|}
\hline Codigo Clon & $\begin{array}{c}\text { Plantas } \\
\text { Establecidas }\end{array}$ & $\begin{array}{l}\text { Plantas } \\
\text { Vivas }\end{array}$ & $\begin{array}{l}\text { SUP } \\
\text { (\%) }\end{array}$ & $\begin{array}{l}\text { ALT } \\
\text { (cm) }\end{array}$ & $\begin{array}{l}\text { DAC } \\
(\mathrm{mm})\end{array}$ & $\begin{array}{c}E \\
\left(\mathrm{~cm}^{3}\right)\end{array}$ \\
\hline 9 Tantehue & 16 & 12 & 75,0 & 180 & 19,5 & 513 \\
\hline 3 Tantehue & 16 & 14 & 87,5 & 170 & 18,4 & 504 \\
\hline 31 Tantehue & 26 & 18 & 69,2 & 170 & 19,3 & 438 \\
\hline 43 Longotoma & 20 & 18 & 90,0 & 120 & 18,3 & 362 \\
\hline 1 Tantehue & 32 & 22 & 68,8 & 140 & 18,5 & 330 \\
\hline Promedio* & 224 & 118 & 52,7 & 150 & 18,4 & 268 \\
\hline 29 Tantehue & 16 & 8 & 50,0 & 150 & 17,9 & 240 \\
\hline 11 Tantehue & 16 & 5 & 31,3 & 180 & 20,2 & 230 \\
\hline 50 Tantehue & 16 & 5 & 31,3 & 160 & 18,8 & 177 \\
\hline 6 Tantehue & 16 & 8 & 50,0 & 140 & 15,8 & 175 \\
\hline 21 Tantehue & 12 & 5 & 41,7 & 140 & 15,8 & 146 \\
\hline 5 Tantehue & 16 & 2 & 12,5 & 130 & 19,5 & 62 \\
\hline 27 Tantehue & 10 & 1 & 10,0 & 160 & 17,0 & 46 \\
\hline 44 Longotoma & 12 & 0 & & & & 0 \\
\hline
\end{tabular}

Cuadro $\mathrm{N}^{\circ} 14$

PARAMETROS MEDIOS EN ENSAYO DE CLONES DE Eucalyptus camaldulensis PULLALLY - EVALUADO A LA EDAD DE 10 MESES

\begin{tabular}{|c|c|c|c|c|c|c|}
\hline Codigo Clon & \begin{tabular}{|c|} 
Plantas \\
Establecidas
\end{tabular} & $\begin{array}{c}\text { Plantas } \\
\text { Vivas }\end{array}$ & $\begin{array}{l}\text { SUP } \\
(\%)\end{array}$ & $\begin{array}{l}\text { ALT } \\
(\mathrm{cm})\end{array}$ & $\begin{array}{c}\text { DAC } \\
(\mathrm{mm})\end{array}$ & $\begin{array}{c}E \\
\left(\mathrm{~cm}^{3}\right)\end{array}$ \\
\hline 9 Tantehue & 16 & 14 & 87,5 & 150 & 16,0 & 336 \\
\hline 29 Tantehue & 16 & 16 & 100,0 & 120 & 15,2 & 277 \\
\hline 31 Tantehue & 16 & 14 & 87,5 & 140 & 13,5 & 223 \\
\hline 43 Longotoma & 16 & 14 & 87,5 & 110 & 15,0 & 217 \\
\hline Promedio* & 160 & 146 & 91,3 & 120 & 13,0 & 185 \\
\hline 3 Tantehue & 16 & 14 & 87,5 & 120 & 12,6 & 167 \\
\hline 1 Tantehue & 32 & 32 & 100,0 & 110 & 12,0 & 158 \\
\hline 5 Tantehue & 16 & 14 & 87,5 & 110 & 12,2 & 143 \\
\hline 11 Tantehue & 16 & 14 & 87,5 & 110 & 11,1 & 119 \\
\hline 50 Tantehue & 16 & 14 & 87,5 & 110 & 10,8 & 112 \\
\hline
\end{tabular}




\section{Cuadro $\mathrm{N}^{\circ} 15$ \\ PARAMETROS MEDIOS EN ENSAYO DE CLONES DE Eucalyptus camaldulensis \\ EL TANGUE - EVALUADO A LA EDAD DE 10 MESES}

\begin{tabular}{|c|c|c|c|c|c|c|}
\hline Codigo Clon & $\begin{array}{c}\text { Plantas } \\
\text { Establecidas }\end{array}$ & $\begin{array}{c}\text { Plantas } \\
\text { Vivas }\end{array}$ & $\begin{array}{l}\text { SUP } \\
(\%)\end{array}$ & $\begin{array}{l}\text { ALT } \\
\text { (cm) }\end{array}$ & $\begin{array}{l}\text { DAC } \\
(\mathrm{mm})\end{array}$ & $\begin{array}{c}E \\
\left(\mathrm{~cm}^{3}\right)\end{array}$ \\
\hline 44 Longotoma & 4 & 2 & 50,0 & 80 & 12,5 & 63 \\
\hline 50 Tantehue & 28 & 11 & 39,3 & 90 & 11,9 & 50 \\
\hline 31 Tantehue & 44 & 18 & 40,9 & 90 & 10,6 & 41 \\
\hline 3 Tantehue & 51 & 22 & 43,1 & 90 & 10,3 & 41 \\
\hline 5 Tantehue & 14 & 5 & 35,7 & 80 & 11,8 & 40 \\
\hline 29 Tantehue & 24 & 8 & 33,3 & 80 & 11,0 & 32 \\
\hline Promedio* & 308 & 110 & 35,7 & 80 & 10,5 & 31 \\
\hline 43 Longotoma & 64 & 24 & 37,5 & 70 & 10,0 & 26 \\
\hline 9 Tantehue & 7 & 2 & 28,6 & 80 & 10,0 & 23 \\
\hline 27 Tantehue & 12 & 2 & 16,7 & 80 & 12,0 & 19 \\
\hline 11 Tantehue & 24 & 7 & 29,2 & 70 & 9,4 & 18 \\
\hline 1 Tantehue & 36 & 9 & 25,0 & 70 & 9,1 & 14 \\
\hline
\end{tabular}

Experiencias anteriores de INFOR indican que la supervivencia de ensayos clonales es inferior a la observada en ensayos con plantas de semillas en Eucalyptus camaldulensis (Gutiérrez y Chung, 1993). Esta diferencia parece obedecer a las condiciones más favorables en que se establecieron los ensayos con plantas de semilla, no obstante, al comparar la supervivencia del ensayo clonal Pullally $(91,3 \%)$ con la reportada para un ensayo establecido en el mismo sitio con plantas obtenidas sexualmente, se obtienen resultados muy similares.

En términos de crecimiento inicial, los clones se comparan favorablemente con plantas obtenidas por propagación sexual. A los 10 meses de edad los ensayos clonales presentaron una altura media de 1,2 m y un diámetro de cuello (DAC) de 13,9 mm, mientras que plantas de semilla, establecidas en un sitio equivalente al de Pullally (sector Longotoma), exhibían a los dos años de edad una altura media de 1,16 m y un DAC medio de 18 mm (Gutiérrez y Chung, 1993).

La mayor tasa de supervivencia (91,3\%) se obtuvo en el ensayo Pullally, mientras que el mayor crecimiento se logró en el ensayo Cuz Cuz. En el ensayo de El Tangue, tanto las variables de crecimiento como la supervivencia, exhiben sus valores más bajos.

El clon con mejor desempeño promedio en los tres ensayos corresponde al $N^{\circ}$ 9, que exhibe simultáneamente los valores más altos de supervivencia, altura y DAC. A nivel de ensayos individuales se observa cierta variación; en Cuz Cuz este mismo clon vuelve a ser el más eficiente, mientras que en El Tangue su desempeño no supera al promedio del ensayo en ninguna de las tres variables evaluadas. Por su parte, en Pullally muestra los mejores valores de crecimiento, pero su supervivencia es inferior al promedio del ensayo.

El menor desempeño promedio lo exhibe el clon $N^{\circ} 44$, el cual fue representado sólo en 
dos ensayos, en Cuz Cuz experimentó una mortalidad completa, mientras que en el Tangue su supervivencia y crecimiento en DAC fueron los mejores del ensayo, siendo su crecimiento en altura intermedio entre el de los restantes clones evaluados en este sitio.

\section{CONCLUSIONES}

Eucalyptus camaldulensis es una especie reconocida por su capacidad de prosperar y producir cosechas aceptables en suelos relativamente pobres y con estación seca prolongada. Estas características le han permitido establecerse con éxito en zonas mediterráneas de distintas partes del mundo y estar relativamente difundida en zonas áridas de Chile.

Los resultados derivados del análisis a nivel de especies en el ensayo Caracas permiten concluir que además de Eucalyptus camaldulensis existen otras especies que presentan el mismo desempeño en sus etapas iniciales de desarrollo, cuando las condiciones de aridez no son tan severas.

Considerando que Eucalyptus cladocalyx, E. sideroxylon y E. tereticornis muestran un desempeño equivalente al de E. camaldulensis, en zonas semiáridas con influencia costera, se puede afirmar que estas especies pueden ofrecen un potencial similar para establecer plantaciones en las zonas áridas del país que están influenciadas por esta condición.

Por el contrario, cuando las condiciones de aridez se acentúan, los resultados de los ensayos Tunga Norte y Huentelauquén indican que la mayoría de las especies evaluadas, con excepción de Eucalypus cladocalyx y Corymbia maculata, pierden competitividad.

La evaluación de los tres ensayos anteriores permite concluir que Eucalyptus cladocalyx es una especie cuyo comportamiento en zonas áridas es similar y en algunos casos superior al de E. camaldulensis, constituyéndose en una de las especies más adecuadas para efectuar plantaciones en estas zonas.

La variabilidad observada entre progenies de las especies anteriores ofrece la posibilidad de selección y mejoramiento genético para incrementar su productividad y desarrollar líneas de mejor adaptación y producción para condiciones locales de cultivo. Estos esfuerzos son especialmente justificados para las especies Eucalyptus camaldulensis, E. cladocalyx y Corymbia maculata.

Respecto de los clones de Eucalyptus camaldulensis, su desempeño en terreno, en términos de crecimiento inicial, es mejor que el observado en plantas de semillas. Sin embargo, los valores de supervivencia resultaron en general inferiores a los reportados para plantas de origen sexual.

La evaluación efectuada resulta demasiado inicial para establecer comparaciones válidas entre clones. 


\section{REFERENCIAS}

Alvear, C. y Gutiérrez, B. 1995. Crecimiento hasta los 42-44 meses de edad y estimación de parámetros genéticos de 23 procedencias y 196 familias de Eucalyptus camaldulensis Dehn en cuatro sitios de la zona central de Chile. Ciencia e Investigación Forestal 9(1): 23-46.

Barros, Santiago, 2009. Álamos y Sauces, Las Salicáceas en el Mundo y en Chile. En Ciencia e Investigación Forestal. Vol $15 \mathrm{~N}^{\circ} 2$. Instituto Forestal. Chile.

Gutiérrez, B. y Chung, P., 1993. Crecimiento inicial de 23 procedencias y 196 familias de Eucalyptus camaldulensis Denh en cuatro sitios de la zona central de Chile. Ciencia e Investigación Forestal 7(1): 5-22.

Gutiérrez, B. y Molina, M., 2008. Selección, multiplicación y evaluación inicial de clones de Eucalyptus camaldulensis para las zonas áridas y semiáridas de Chile. Ciencia e Investigación Forestal 14(1).

INFOR, 2010. Anuario Forestal, Boletín Estadístico N²128. Instituto Forestal. Chile.

Smith, N., 1997. Comportamiento de Eucalyptus camaldulensis en Longotoma, Chile. Base para el mejoramiento de la especie en la zona semiárida. En: Valdebenito, G y Benedetti, S. (editores). Forestación y Silvicultura en Zonas Áridas y Semiáridas de Chile. INFOR-CORFO. Santiago, Chile. Pp: 186-196. 